\title{
Scale similarity of MHD turbulence in the Earth's core
}

\author{
Masaki Matsushima \\ Department of Earth and Planetary Sciences, Tokyo Institute of Technology, Tokyo 152-8551, Japan
}

(Received November 19, 2003; Revised May 25, 2004; Accepted May 25, 2004)

\begin{abstract}
Turbulent motions in the core, being highly anisotropic because of the influence of the Earth's rotation and its magnetic field, cause the eddy diffusion of large-scale fields much more effectively than the molecular diffusion. Reliable estimates of the eddy diffusivities, or the subgrid-scale fluxes, are therefore of significance. In this paper, scale similarity of magnetohydrodynamic turbulence in a rapidly rotating system is investigated to model subgrid-scale processes, as used in large-eddy simulations. The turbulent flux has been computed by taking an ensemble average of results of direct numerical simulations, which are to be employed in this paper, over the computational box which represents a small region in the Earth's core. The anisotropy of turbulent flux computed after averaging over segments into which the box is divided remains unchanged even when the size of segments changes. Dependence of turbulent flux computed from fields to which a spatial filter is applied on its width indicates that subgrid-scale flux can be evaluated through extrapolation. This method will be useful for performing global geodynamo simulations taking into account subgrid-scale processes.
\end{abstract}

Key words: MHD turbulence, Earth's core, subgrid scale, scale similarity.

\section{Introduction}

The geodynamo, which is a generation mechanism of the Earth's magnetic field, has been understood through threedimensional self-consistent magnetohydrodynamic (MHD) dynamo simulations in rapidly rotating spherical shells (e.g. Roberts and Glatzmaier, 2000; Kono and Roberts, 2002). For the first stage, it was of significance to examine basic physical processes of magnetic field generation and structures of the velocity and the magnetic fields; for example, columnar convection cells parallel to the rotational axis occur in a rapidly rotating spherical shell, and convergence of flows into those with negative axial vorticity near the equatorial plane works upon confinement of the magnetic field there. Helical flows within such convection cells are very important for the magnetic field to be generated through advection and stretch of the field (e.g. Kageyama et al., 1995; Kageyama and Sato, 1997; Olson et al., 1999; Takahashi et al., 2001). In the meantime, systematic survey of MHD dynamo behavior was made (Christensen et al., 1999), and furthermore even a dynamo benchmark, which provides a standard solution of a three-dimensional, self-consistent spherical shell MHD dynamo model, was constructed (Christensen et al., 2001).

Non-dimensional parameters used in numerical simulations, however, have been very far from those of the real Earth. For instance, the Ekman number, $E=v / 2 \Omega D^{2}$, where $v$ is the kinematic viscosity, $D$ the thickness of a rotating spherical shell, and $\Omega$ its angular velocity, ranges from $O\left(10^{-1}\right)$ to $O\left(10^{-6}\right)$, although $E$ of the Earth's core is of the order of $10^{-15}\left(v=10^{-6} \mathrm{~m}^{2} / \mathrm{s}, D=2.27 \times 10^{6} \mathrm{~m}\right.$, and

Copy right (c) The Society of Geomagnetism and Earth, Planetary and Space Sciences (SGEPSS); The Seismological Society of Japan; The Volcanological Society of Japan The Geodetic Society of Japan; The Japanese Society for Planetary Sciences; TERRAPUB.
$\left.\Omega=7.29 \times 10^{-5} \mathrm{rad} / \mathrm{s}\right)$. No one knows what arises from the obvious difference. To understand the realistic Earth's dynamo, much smaller Ekman numbers and much larger Rayleigh numbers must be adopted in numerical simulations.

In reality, because of very small molecular diffusivities, it is likely that the Earth's liquid core is in a turbulent state; that is, a range of spatial scales of convective motions in the core is very broad, from global core scales to dissipative scales. Core turbulence is also expected to be highly anisotropic because of the influence of the Earth's rotation and its magnetic field (e.g. Braginsky and Meytlis, 1990; Matsushima et al., 1999; Roberts and Glatzmaier, 2000). Such anticipated turbulent motions can never be ignored, since large-scale fields are diffused much more effectively by anisotropic turbulent eddies than by molecular diffusive processes. At the present, however, it is impossible to carry out global geodynamo simulations taking into account molecular diffusivities because of limited spatial resolution and computational power. Instead, the expedient of using eddy diffusivities has usually been adopted, which is one of methods to parameterize physical processes where the unresolved subgrid scales (SGS) are involved. Artificial hyper-diffusivities, which work more effectively upon smaller-scale fields, were also used for very small $E$ (e.g. Glatzmaier and Roberts, 1995a, b), although it was pointed out that they can alter the essential features in MHD dynamo simulations (e.g. Zhang and Jones, 1997). In this sense global geodynamo simulations carried out so far have aimed at examining the evolution of large-scale fields alone, and can be regarded as a kind of large-eddy simulation.

In large-eddy simulation (LES), which is known as a turbulence simulation method, a spatial function is used to eliminate small-scale components, or SGS components, that contribute to the energy dissipation. It is then required to model 
their effects on large-scale components. In numerical calculations of magneto-convection in a rapidly rotating system, Buffett (2003) examined four SGS models for LES; the eddy diffusivity model, the hyper-diffusivity model, the Smagorinsky model, and the similarity model. In the eddy diffusivity model, spatially constant eddy diffusivities were defined on the basis of the characteristic length and velocity. In the hyper-diffusivity model, the diffusivities were increased with decrease in the length-scale. In the Smagorinsky model, the diffusivities were expressed in terms of the characteristic length-scale and the strain-rate tensor. These three models are all based on eddy diffusivities. In the similarity model, as provided by Bardina et al. (1980) and simplified later by Germano (1986), the unknown SGS flux is approximated by terms calculated from the resolved field to which an additional spatial filter is applied. In fact, the scale similarity model is based on two assumptions; one is that components of the maximum subgrid-scale are similar to those of the minimum grid-scale, and the other is that subgrid-scale components immediately below the minimum grid-scale have the greatest influence on those of the gridscale. Buffett (2003) concluded that the similarity model was much more successful in reproducing the anisotropy in the SGS estimates. However the constants in the scale-similarity model were determined by referencing to those of direct numerical simulations (DNS) with LES. We need a better evaluation of the constants.

We investigate in this paper the scale similarity of MHD turbulence in the core, aiming at finding a simple method to evaluate the SGS flux which can be anisotropic. We use results of DNS of magneto-convective turbulence in a rapidly rotating system carried out by Matsushima (2001), who attempted to model subgrid-scale processes like turbulent heat flux. We examine the dependence of turbulent fluxes on a grid scale and a filter width by taking an ensemble average over the whole computational box for DNS. Such a relation will be helpful to carry out numerical simulations taking into account SGS processes.

\section{Results of Direct Numerical Simulations}

We have been performing direct numerical simulations in a rapidly rotating system to examine the MHD turbulence possibly taking place in the Earth's core. To represent a very small region in the fluid outer core, a rectangular box with periodic boundaries is adopted, and its location is defined by the direction of gravity, or the colatitude, $\lambda$, in the core. A uniform magnetic field has been imposed to represent a large-scale magnetic field generated by dynamo processes. Turbulent motions are driven by large-scale buoyancy field, or a temperature gradient parallel to the direction of gravity. Local Cartesian coordinates, $(x, y, z)$, are defined; the $z$ coordinate is aligned in the direction of the rotation axis, and the $y$-coordinate in the azimuthal direction (Fig. 1). Then basic equations have been solved by using a control volume method (Matsushima et al., 1999).

Scaling length by $a$ (the size of the computational box in the $z$-direction), time by $a^{2} / \eta$, magnetic field by $B_{0}$, pressure by $2 \Omega \eta \rho$, and temperature by $\beta a$, where $\eta$ is the magnetic diffusivity, $\rho$ the density of core fluid, and $\beta$ the large-scale buoyancy gradient, the basic equations to be solved are given
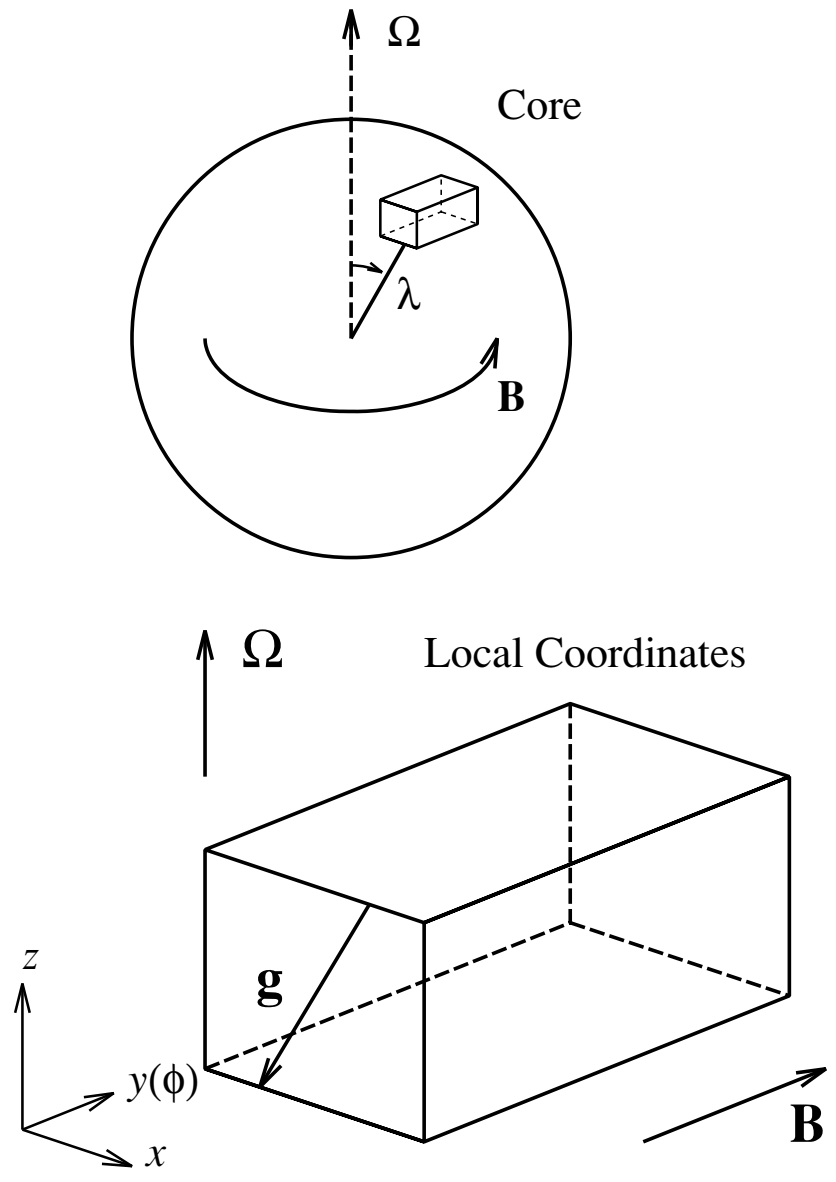

Fig. 1. Schematic pictures of the computational box and its relation to the Earth's core.

as

$$
\begin{aligned}
& \varepsilon_{\eta}\left\{\partial_{t} \boldsymbol{v}+(\boldsymbol{v} \cdot \nabla) \boldsymbol{v}\right\}=-\nabla p-\mathbf{1}_{z} \times \boldsymbol{v} \\
&+\Lambda(\nabla \times \boldsymbol{b}) \times\left(\mathbf{1}_{B}+\boldsymbol{b}\right) \\
&+R a \theta \mathbf{1}_{r}+\varepsilon_{v} \nabla^{2} \boldsymbol{v}, \\
& \partial_{t} \boldsymbol{b}+(\boldsymbol{v} \cdot \nabla) \boldsymbol{b}=\left\{\left(\mathbf{1}_{B}+\boldsymbol{b}\right) \cdot \nabla\right\} \boldsymbol{v}+\nabla^{2} \boldsymbol{b}, \\
& \partial_{t} \theta+\boldsymbol{v} \cdot \nabla \theta=-\boldsymbol{v} \cdot \nabla \bar{\Theta}+P q \nabla^{2} \theta, \\
& \nabla \cdot \boldsymbol{v}=0, \quad \nabla \cdot \boldsymbol{b}=0,
\end{aligned}
$$

where $\boldsymbol{v}$ is the velocity field in a coordinate system rotating with angular velocity, $\boldsymbol{\Omega}=\boldsymbol{\Omega} \mathbf{1}_{z}, \mathbf{1}_{z}$ the unit vector in the $z$-direction, $\boldsymbol{b}$ the magnetic field, $p$ the reduced pressure, and $\theta$ the temperature deviated from a large-scale field, $\bar{\Theta}$ (a variable with an overbar is a mean field). Nondimensional numbers appeared in these equations are the magnetic Ekman number, $\varepsilon_{\eta}=\eta / 2 \Omega a^{2}$, the Elsasser number, $\Lambda=B_{0}^{2} \sigma / 2 \Omega \rho$, the modified Rayleigh number, $R a=$ $\alpha g \beta a^{2} / 2 \Omega \eta$, the Ekman number, $\varepsilon_{v}=v / 2 \Omega a^{2}$, and the Roberts number, $P q=\kappa / \eta$, where $\sigma$ is the electrical conductivity, $\alpha$ the coefficient of thermal expansion, $g$ the gravity, and $\kappa$ the thermal diffusivity. We have examined the effect of the turbulent heat transport, $\overline{v \cdot \nabla \theta}$, on the mean field, $\bar{\Theta}$. Using (4), we have $\overline{\boldsymbol{v} \cdot \nabla \theta}=\nabla \cdot \overline{\theta \boldsymbol{v}}=\nabla \cdot \overline{\boldsymbol{I}}$, where 

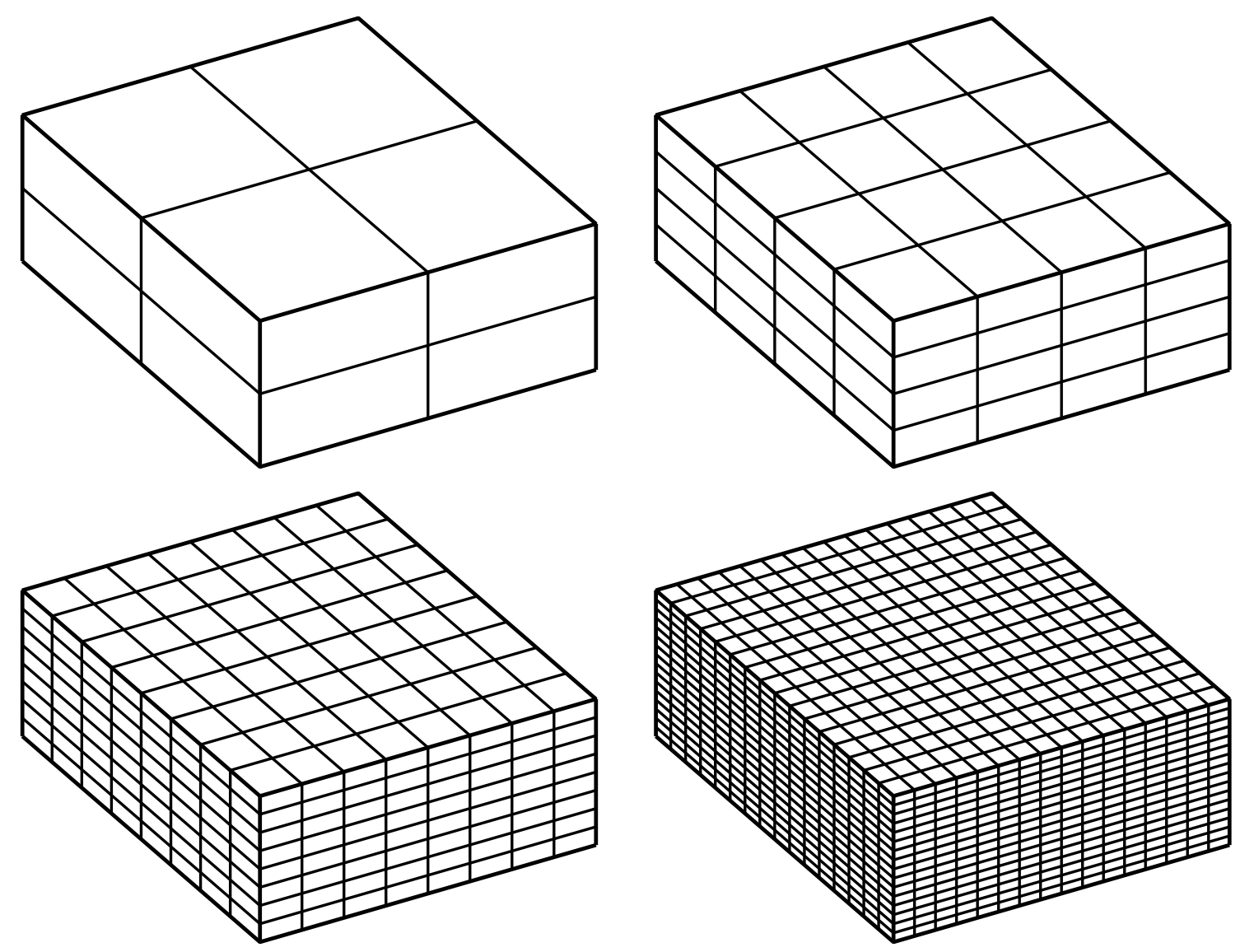

Fig. 2. The computational box divided into $\left(2^{n}\right)^{3}$ segments, where $n=1,2,3$, and 4 .

$\overline{\boldsymbol{I}}=\overline{\theta \boldsymbol{v}}$ is the turbulent heat flux; the flux of heat created by the turbulent motions. Results of numerical simulations are summarized in Matsushima et al. (1999) and Matsushima (2001). In the following, we investigate the scale similarity using the results thus obtained so far.

\section{Investigation of Scale Similarity}

\subsection{Dependence on grid scale}

We have considered the computational box as a very small region in the core; that is, its size may correspond to a grid spacing for a global geodynamo simulation. We have evaluated the turbulent heat flux, $\boldsymbol{I}$, by taking a volume integral over the computational region of volume $\mathcal{V}$ as

$$
\overline{\boldsymbol{I}}=\overline{\theta v}=\frac{1}{\mathcal{V}} \int \theta \boldsymbol{v} d \mathcal{V} .
$$

To investigate the scale similarity, we divide the computational region into $\left(2^{n}\right)^{3}$ parts, where $n=1,2,3,4$, and 5 , as shown in Fig. 2 (a view for $n=5$ is not illustrated). We first take ensemble averages over these segments, and obtain mean values, $\breve{\theta}$ and $\breve{v}$, which are regarded as representatives for respective segments. Then using $\breve{\theta}$ and $\breve{v}$ thus obtained, we calculate the turbulent heat flux, $\overline{\boldsymbol{I}}^{(n)}=\breve{\breve{\theta} \breve{\boldsymbol{v}}}$, for $n=1$ to $n=5$. We here define the relative grid scale as, for example, $l_{x}^{(n)} / L_{x}$, where $l_{x}^{(n)}$ and $L_{x}$ are lengths of a segment for certain $n$ and the whole region, respectively, in the $x$-direction. In this study, the number of division is identical in the three directions, and we have $l_{x}^{(n)} / L_{x}=l_{y}^{(n)} / L_{y}=l_{z}^{(n)} / L_{z}=2^{-n}$.

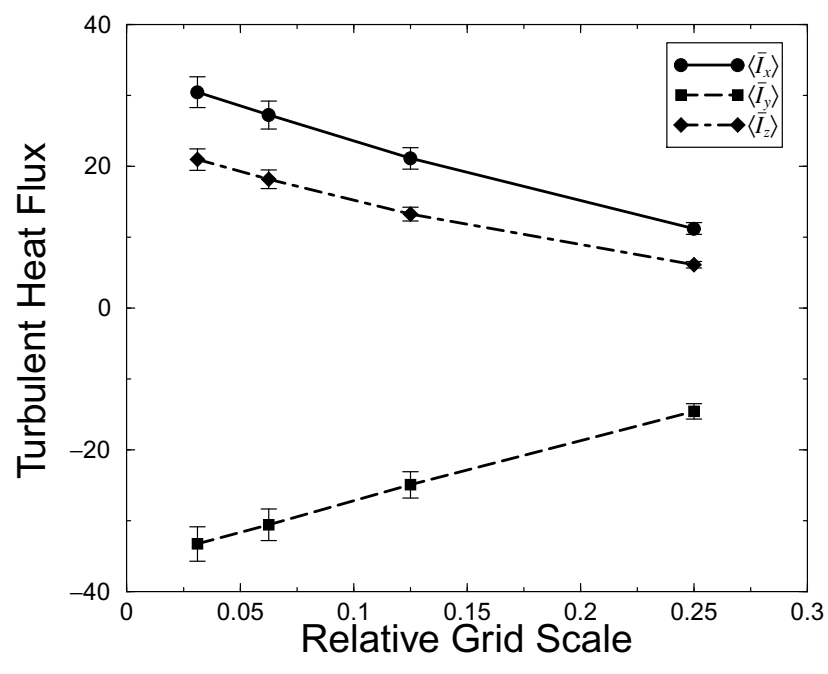

Fig. 3. The relation between the relative grid scale and the turbulent heat flux, $\left(\left\langle\bar{I}_{x}\right\rangle,\left\langle\bar{I}_{y}\right\rangle,\left\langle\bar{I}_{z}\right\rangle\right)$, for $\lambda=\pi / 6, R a=240, \Lambda=10, \varepsilon_{v}=10^{-3}$, $\varepsilon_{\eta}=10^{-2}$, and $P q=10^{-1}$. The error bars represent the standard deviation for temporal averages.

Figure 3 shows the relative grid-scale dependence of the turbulent heat flux, $\left\langle\bar{I}_{x}^{(n)}\right\rangle,\left\langle\bar{I}_{y}^{(n)}\right\rangle$ and $\left\langle\bar{I}_{z}^{(n)}\right\rangle$ for $\lambda=\pi / 6$, $R a=240, \Lambda=10, \varepsilon_{\nu}=10^{-3}, \varepsilon_{\eta}=10^{-2}$, and $P q=10^{-1}$, where \langle\rangle denotes the ensemble average as averages over one magnetic diffusion time. There seems to be a linear relationship between the turbulent heat flux and the relative 


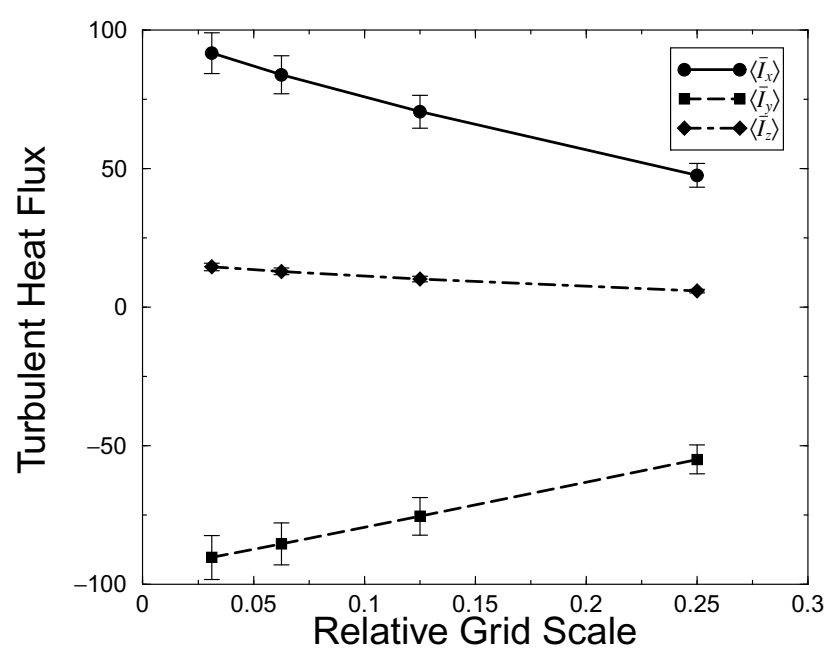

Fig. 4. The relation between the relative grid scale and the turbulent heat flux, $\left(\left\langle\bar{I}_{x}\right\rangle,\left\langle\bar{I}_{y}\right\rangle,\left\langle\bar{I}_{z}\right\rangle\right)$, for $\lambda=\pi / 3, R a=240, \Lambda=10, \varepsilon_{v}=10^{-3}$, $\varepsilon_{\eta}=10^{-2}$, and $P q=10^{-1}$. The error bars represent the standard deviation for temporal averages.

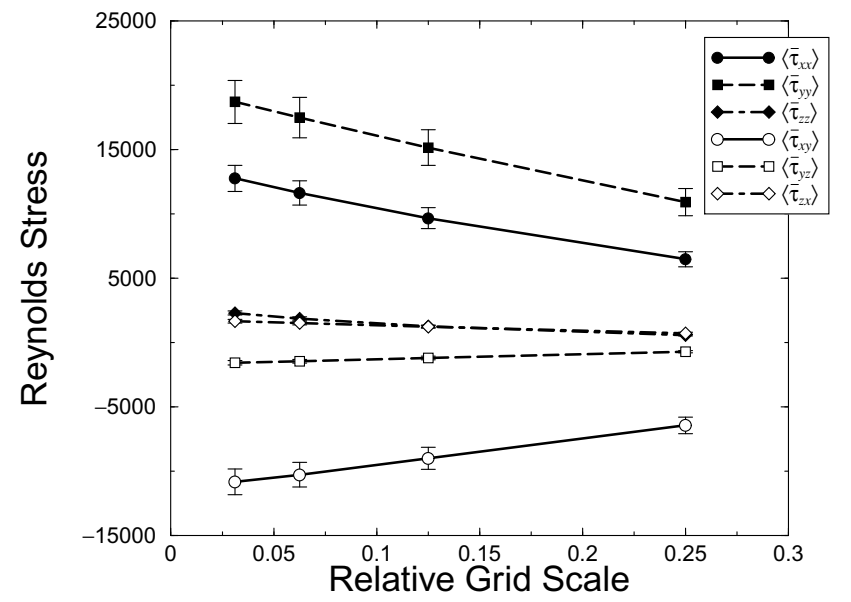

Fig. 5. The relation between the relative grid scale and the turbulent momentum flux, $\left\langle\bar{\tau}_{i j}\right\rangle$, for $\lambda=\pi / 3, R a=240, \Lambda=10, \varepsilon_{v}=10^{-3}$, $\varepsilon_{\eta}=10^{-2}$, and $P q=10^{-1}$. The error bars represent the standard deviation for temporal averages.

grid scale of a segment over which the first ensemble average is taken. The same relationship can also be found for $\lambda=$ $\pi / 3$ (Fig. 4). These results indicate that the direction of the turbulent heat flux remains unchanged for spatial filtering; that is, the anisotropy is conserved. The magnitude, however, decreases by smoothing spatial distribution of fields, $\theta$ and $v$, onto coarser grid points.

We have so far examined the scale similarity of the turbulent heat flux. The characteristics may also be found in the Reynolds stress, $\bar{\tau}_{i j}=\overline{v_{i} v_{j}}$, or the turbulent momentum flux. Hence we calculate $\bar{\tau}_{i j}^{(n)}={\overline{v_{i}}}_{\breve{v}_{j}}$ in the same manner. Figure 5 shows the relative grid-scale dependence of the Reynolds stress, $\left\langle\bar{\tau}_{i j}^{(n)}\right\rangle$, for $\lambda=\pi / 3, R a=240, \Lambda=10$, $\varepsilon_{v}=10^{-3}, \varepsilon_{\eta}=10^{-2}$, and $P q=10^{-1}$. It should be remarked that a similar relationship is found between all the components of $\tau_{i j}$ and the relative grid-scale.

\subsection{Dependence on filter width}

In LES, as described above, subgrid-scale components are eliminated by using a spatial filter as

$$
\widehat{\boldsymbol{v}}(\boldsymbol{r}, t)=\int \widehat{G}\left(\boldsymbol{r}-\boldsymbol{r}^{\prime}\right) \boldsymbol{v}\left(\boldsymbol{r}^{\prime}, t\right) d \boldsymbol{r}^{\prime},
$$

where $\widehat{G}$ is a filter function. Upon applying the filter function to the energy equation, without a large-scale temperature gradient, for example,

$$
\partial_{t} \theta+\boldsymbol{v} \cdot \nabla \theta=P q \nabla^{2} \theta
$$

one obtains that for large-scale fields,

$$
\partial_{t} \widehat{\theta}+\widehat{\boldsymbol{v}} \cdot \nabla \widehat{\theta}=P q \nabla^{2} \widehat{\theta}-\nabla \cdot \boldsymbol{I}^{*},
$$

where $I^{*}$ denotes the SGS heat flux given as

$$
I^{*}=\widehat{\theta v}-\widehat{\theta} \widehat{\boldsymbol{v}} .
$$

The scale similarity model is based on scale invariance that certain features of the fields remain the same in different scales; that is, the structure of fields at subgrid scales is postulated to be similar to that at grid scales. Hence approximation of $\boldsymbol{I}^{*}$ in the similarity model of Germano (1986) is given as

$$
I^{*}=C_{I}(\widetilde{\hat{\theta}} \widehat{\boldsymbol{v}}-\widetilde{\widehat{\theta}} \widetilde{\boldsymbol{v}})
$$

where $C_{I}$ is a constant and the tilde denotes a wider filter. Similarly, the SGS momentum flux, $\tau_{i j}^{*}$, is given as

$$
\tau_{i j}^{*}=C_{\tau}\left({\widetilde{v_{i}}}_{\widehat{v}_{j}}-{\widetilde{\widehat{v}_{i}}}_{i} \widetilde{\widehat{v}}_{j}\right)
$$

where $C_{\tau}$ is another constant.

There are many studies, in which the scale-similarity model is proposed and used, although only the SGS stress tensor is modeled in most of them. For example, expansion of the grid-scale velocity field to subgrid-scales in spectral space yields an estimation of a velocity field with unresolved scales (Domaradzki and Saiki, 1997). This procedure is extended to that in physical space (Domaradzki and Loh, 1999). In these models a grid-scale field is expanded to that with subgrid-scales which are two times smaller than the smallest resolved scale. In the meantime, Horiuti (1997) proposed a new dynamic subgrid-scale mixed model, in which an improved Bardina model as a scale-similarity model was linearly combined with the Smagorinsky model, for the SGS Reynolds stress.

It should be pointed out that the anisotropy of the SGS fluxes of both heat and momentum can be reproduced by the scale-similarity model, but not by the SGS models in terms of scalar eddy diffusivities (Buffett, 2003). It is probable that the turbulent eddies in the Earth's core are highly anisotropic due to the influence of the Earth's rotation and the strong magnetic field, and that they result in the turbulent fluxes which are highly anisotropic also (Braginsky and Meytlis, 1990; St. Pierre, 1996; Matsushima et al., 1999). In this sense, the scale-similarity model is appropriate to the SGS fluxes of heat and momentum in rotating magneto-convective turbulence. Buffet (2003), however, determines the constants 
$C_{I}$ and $C_{\tau}$ in (10) and (11), respectively, to adjust the amplitude of the predicted fluxes of heat and momentum to those obtained from DNS. Some method of determining the constants or alternative one is therefore required.

The results obtained so far in this study, as shown in Figs. 3-5, suggest that the scale similarity model in terms of the relative grid scale is helpful in estimating the subgrid scales. Keeping the scale similarity in mind, the computational region has been divided into segments, whose size has been presumed to be subgrid scales. In reality, however, the minimum length scale in numerical simulations corresponds to the grid spacing, and a finer one is not defined. Moreover, the relationship between the turbulent flux and the relative grid scale must hold good not only on the temporal average but also every moment. Hence, as done by Germano (1986), we apply a spatial filter to the grid-scale fields using a filter width larger than the grid spacing. Then the subgrid-scale fields are to be estimated. In this study, we use a top-hat filter whose function is defined as

$$
\widetilde{G}(\boldsymbol{r})=\widetilde{G}(x, y, z)=\left\{\begin{aligned}
\frac{1}{\Delta_{x}^{*} \Delta_{y}^{*} \Delta_{z}^{*}} & \\
& \text { for }|x|<\frac{\Delta_{x}^{*}}{2} \\
& |y|<\frac{\Delta_{y}^{*}}{2} \\
& |z|<\frac{\Delta_{z}^{*}}{2}, \\
0 & \text { otherwise, }
\end{aligned}\right.
$$

where $\Delta_{x}^{*}, \Delta_{y}^{*}$ and $\Delta_{z}^{*}$ are filter widths in the $x$-, $y$ - and $z$ directions, respectively. We apply top-hat filters with various widths to $\theta$ and $v$ obtained through DNS as

$$
\widetilde{\theta}^{\langle 2 n+1\rangle}(\boldsymbol{r}, t)=\int \widetilde{G}^{\langle 2 n+1\rangle}\left(\boldsymbol{r}-\boldsymbol{r}^{\prime}\right) \theta\left(\boldsymbol{r}^{\prime}, t\right) d \boldsymbol{r}^{\prime},
$$

where $\widetilde{G}^{\langle 2 n+1\rangle}$ is given by (12) with $\Delta_{x}^{*}=(2 n+1) \Delta_{x}$, $\Delta_{y}^{*}=(2 n+1) \Delta_{y}$ and $\Delta_{z}^{*}=(2 n+1) \Delta_{z} ; \Delta_{x}, \Delta_{y}$ and $\Delta_{z}$ are grid spacings in the $x$-, $y$ - and $z$-directions, respectively. The turbulent heat flux and the turbulent momentum flux are computed by taking ensemble average over the whole computational region, as $\overline{\boldsymbol{I}}^{\langle 2 n+1\rangle}=\widetilde{\widetilde{\theta}^{\langle 2 n+1)} \widetilde{\boldsymbol{v}}^{(2 n+1)}}$ and $\bar{\tau}_{i j}^{\langle 2 n+1\rangle}=\overline{\widetilde{v}_{i}^{\langle 2 n+1\rangle} \widetilde{v}_{j}^{\langle 2 n+1\rangle}}$, respectively. We then investigate dependence of these turbulent fluxes on the filter width at a certain time.

Figures 6 and 7 show dependence of the turbulent heat flux and the turbulent momentum flux, respectively, on the filter width in units of the grid spacing for $\lambda=\pi / 3, R a=$ 240, $\Lambda=10, \varepsilon_{v}=10^{-3}, \varepsilon_{\eta}=10^{-2}$, and $P q=10^{-1}$ at a certain time. As seen in these figures, the turbulent fluxes approach to zero as the filter width becomes large, and do not vary linearly. Nevertheless, these variations can be approximated to straight lines as far as filter widths are small. Hence $\overline{\boldsymbol{I}}^{\langle 0\rangle}$, which may correspond to the turbulent heat flux to be evaluated, can be derived from extrapolation as $\overline{\boldsymbol{I}}^{\langle 0\rangle}=\left(3 \overline{\boldsymbol{I}}^{\langle 1\rangle}-\overline{\boldsymbol{I}}^{\langle 3\rangle}\right) / 2$, provided that the relation can be extended to zero filter width.

\section{Discussion}

Matsushima (2001) attempted to determine geophysically realistic diffusivity tensors for use in global geodynamo sim-

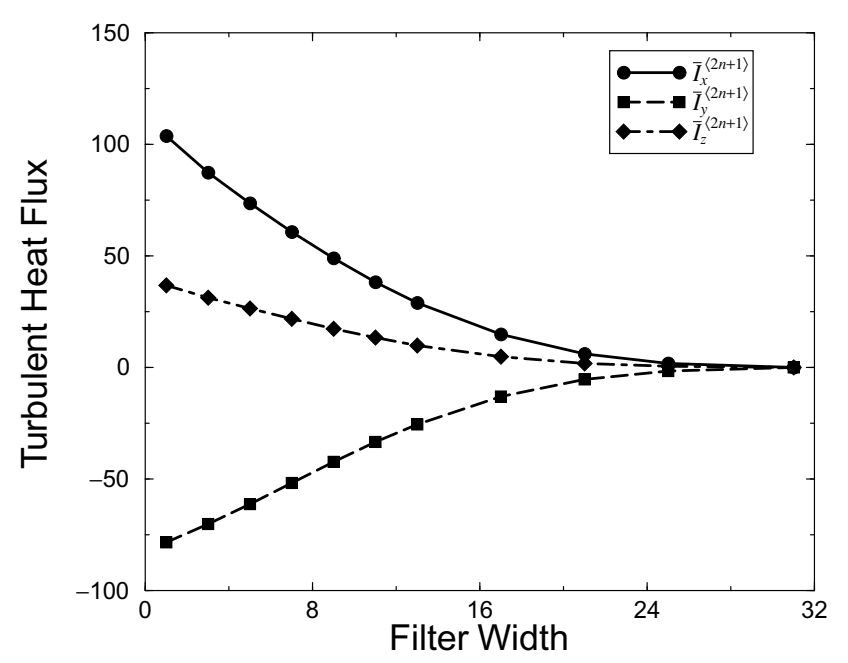

Fig. 6. The relation between the filter width and the turbulent heat flux, $\left(\bar{I}_{x}^{\langle 2 n+1\rangle}, \bar{I}_{y}^{\langle 2 n+1\rangle}, \bar{I}_{z}^{\langle 2 n+1\rangle}\right)$, for $\lambda=\pi / 3, R a=240, \Lambda=10, \varepsilon_{v}=10^{-3}$, $\varepsilon_{\eta}=10^{-2}$, and $P q=10^{-1}$ at a certain time.

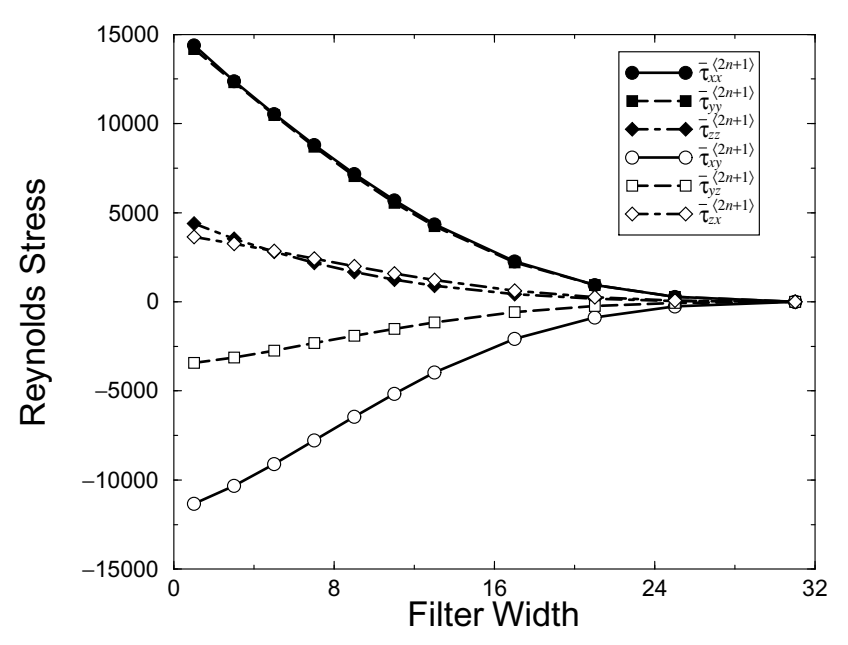

Fig. 7. The relation between the filter width and the turbulent momentum flux, $\bar{\tau}_{i j}^{\langle 2 n+1\rangle}$, for $\lambda=\pi / 3, R a=240, \Lambda=10, \varepsilon_{v}=10^{-3}, \varepsilon_{\eta}=10^{-2}$, and $P q=10^{-1}$ at a certain time.

ulations, and modeled the turbulent heat flux in terms of second moment closure. It was pointed out that the expression of turbulent heat flux gives results that are correct to within an order of magnitude in comparison with those of DNS. In fact, the proposed model seems to be useful in quantifying the effect of anisotropic turbulence on global geodynamo models, since a diffusivity tensor, which is spatially and temporally variable, can be determined from local parameters only. It should be noted, however, that the Reynolds stress obtained through DNS was used to estimate the turbulent heat flux. Thus there remains room for improvement in the model, which has not been used in numerical simulations yet. In the meantime, Phillips and Ivers (2000) developed general expressions of the turbulent diffusion tensor in spherical geometries. Complicated spectral interactions associated with anisotropic viscous and thermal diffusion tensors were further derived for use in spherical MHD dynamo simulations in spectral space for rapid rotation (Phillips and Ivers, 2001) 


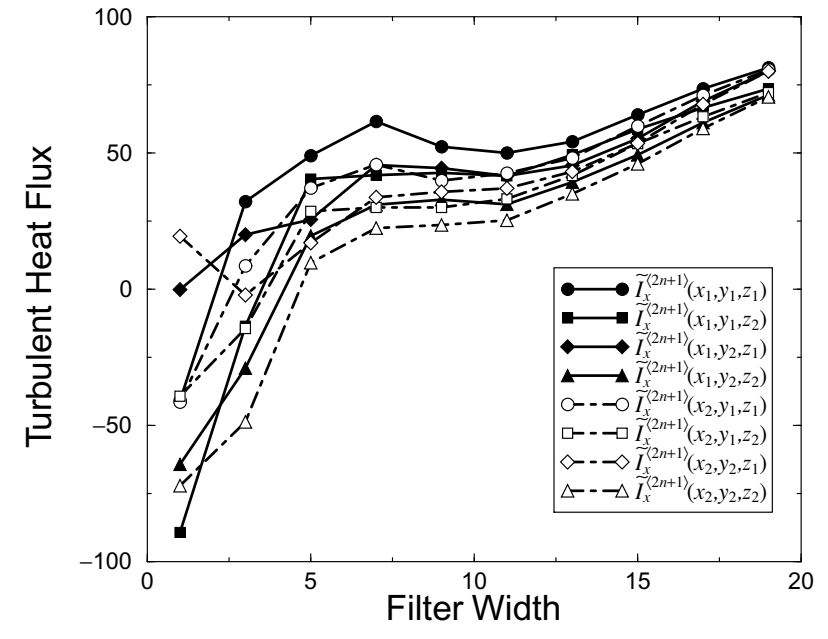

Fig. 8. The relation between the filter width and the $x$-component of the turbulent heat flux, $\widetilde{I}_{x}$, at eight adjacent grid points for $\lambda=\pi / 3$, $R a=240, \Lambda=10, \varepsilon_{v}=10^{-3}, \varepsilon_{\eta}=10^{-2}$, and $P q=10^{-1}$ at a certain time.

and for a strong azimuthal magnetic field (Phillips and Ivers, 2003).

As another model for use in carrying out global geodynamo simulations, the scale similarity was investigated in this paper. The turbulent fluxes as ensemble averages spatially over the computational region and also temporally keep their anisotropic nature unchanged with increase in the relative grid scale of segments, for which $\breve{\theta}$ and $\breve{\boldsymbol{v}}$ are obtained as representatives from $\theta$ and $v$ located within respective segments. The anisotropy is also kept unvaried with increase in the filter width of a top-hat filter, which is applied to $\theta$ and $v$ before calculating the turbulent fluxes as spatial averages over the computational box at a certain time. Magnitude of respective components of the turbulent flux decreases with increase in the filter width, but it is possible to evaluate the turbulent flux by extrapolation, as mentioned in the previous section.

We have so far examined the turbulent flux as a spatial average over the computational box for DNS. We can employ the relation between the turbulent flux and the filter width in global simulations when it holds at respective grid points, $\left(x_{i}, y_{j}, z_{k}\right)$, where $x_{i}=i L_{x} / N_{x}\left(i=0, \cdots, N_{x}\right)$, $y_{j}=j L_{y} / N_{y}\left(j=0, \cdots, N_{y}\right)$, and $z_{k}=k L_{z} / N_{z}(k=$ $\left.0, \cdots, N_{z}\right) . N_{x}, N_{y}$ and $N_{z}$ are the number of grid points in the $x-, y$ - and $z$-directions, respectively. We show, in Fig. 8, dependence of the $x$-component of turbulent heat flux, $\widetilde{I}_{x}=\widetilde{\theta v_{x}}$, on the filter width at eight adjacent grid points for $\lambda=\pi / 3, R a=240, \Lambda=10, \varepsilon_{v}=10^{-3}, \varepsilon_{\eta}=10^{-2}$, and $P q=10^{-1}$. Clearly, values of $\tilde{I}_{x}$ approach to a mean value as the filter width becomes large, whereas they disperse for small filter widths even at adjacent grid points. At seven of eight grid points, $\widetilde{I}_{x}$ monotonously varies between filter width 1 and 5 in units of the grid spacing. Only at one grid point, specifically at $\left(x_{2}, y_{1}, z_{2}\right)$, we have $\widetilde{I}_{x}^{\langle 1\rangle}>\widetilde{I}_{x}^{\langle 3\rangle}$ and $\widetilde{I}_{x}^{\langle 3\rangle}<\widetilde{I}_{x}^{\langle 5\rangle}$. The same was found at about one third of whole grid points. However, the results, as shown in Figs. 6 and 7 , demonstrate that the relation between the turbulent fluxes, averaged over the computational box for DNS, and the filter width can be extended to zero filter width. It is therefore natural to presume that the extrapolation is available at respective grid points. That is, the turbulent flux, or the subgrid-scale flux, at every grid point would be evaluated in the same manner as in the previous section.

In the present method of estimating the turbulent flux, the constants $C_{I}$ and $C_{\tau}$, in (10) and (11), respectively, are not determined, but $\widehat{\theta v}$ and $\widehat{v_{i} v_{j}}$ are directly evaluated. On the other hand, Buffett (2003) compared amplitudes of the SGS fluxes in the similarity model with those in results of DNS, and found that $C_{I}$ and $C_{\tau}$ are of the order of unity. He therefore proposed to use models with $C_{I}=C_{\tau}=1$. However, he also mentioned that the SGS fluxes of heat and momentum are underestimated in the similarity model; that is, $C_{I} \leq 1$ and $C_{\tau} \leq 1$. There is a possibility that this point is improved by using the method proposed in this paper. At the next step, it is required to adopt it in numerical simulations and to confirm it by comparing results.

\section{Conclusions}

We have investigated the scale similarity of MHD turbulence in a rapidly rotating system by using results of direct numerical simulations carried out by Matsushima (2001). The main objective is to find an alternative method of estimating the turbulent flux as subgrid-scale processes. The results are summarized as follows:

1. The direction of turbulent fluxes, averaged both over the computational box after taking average over its segments and over the time, does not depend on the relative grid scale of segments. This suggests that the scale similarity substantially holds good and that the anisotropy of turbulent fluxes remains unchanged for spatial filtering as applied in this paper.

2. Dependence of turbulent fluxes, averaged over the computational box after filtering, on the width of spatial filters indicates that their variations can be approximated to straight lines for small filter width. This implies that SGS turbulent fluxes can be evaluated through simple extrapolation.

3. SGS turbulent fluxes at respective grid points can be evaluated in the same manner as the previous one. They approach to their means over the computational box as the width of spatial filters becomes large.

We need to demonstrate the validity of the method, proposed in the present study, of estimating the SGS fluxes; that is, further investigation is necessary. Nevertheless, the present method would be useful in performing global geodynamo simulations incorporating the SGS physical processes. It can also be applied to numerical simulations of rotating magneto-convective turbulence, as performed by Matsushima et al. (1999) and Matsushima (2001), for much smaller diffusivities.

Acknowledgments. The author thanks the editor, Dr. H. Matsui and an anonymous reviewer for their constructive comments. This work was supported by the Japan Society for the Promotion of Science under the grant-in aid for scientific research No. 15540405. 


\section{References}

Bardina, J., J. H. Ferziger, and W. C. Reynolds, Improved subgrid scale models for large-eddy simulations, Am. Inst. Aeronaut. Astronaut., Paper 80-1357, 1980.

Braginsky, S. I. and V. P. Meytlis, Local turbulence in the Earth's core, Geophys. Astrophys. Fluid Dynam., 55, 71-87, 1990.

Buffett, B. A., A comparison of subgrid-scale models for large-eddy simulations of convection in the Earth's core, Geophys. J. Int., 153, 753-765, 2003.

Christensen, U., P. Olson, and G. A. Glatzmaier, Numerical modelling of the geodynamo: a systematic parameter study, Geophys. J. Int., 138, 393 409, 1999.

Christensen, U. R., J. Aubert, P. Cardin, E. Dormy, S. Gibbons, G. A. Glatzmaier, E. Grote, Y. Honkura, C. Jones, M. Kono, M. Matsushima, A. Sakuraba, F. Takahashi, A. Tilgner, J. Wicht, and K. Zhang, A numerica dynamo benchmark, Phys. Earth Planet. Inter., 128, 25-34, 2001.

Domaradzki, J. A. and K.-C. Loh, The subgrid-scale estimation model in the physical space representation, Phys. Fluids, 11, 2330-2342, 1999.

Domaradzki, J. A. and E. M. Saiki, A subgrid-scale model based on the estimation of unresolved scales of turbulence, Phys. Fluids, 9, 21482164, 1997.

Germano, M., A proposal for a redefinition of the turbulent stresses in the filtered Navier-Stokes equations, Phys. Fluids, 29, 2323-2324, 1986.

Glatzmaier, G. A. and P. H. Roberts, A three-dimensional self-consistent computer simulation of a geomagnetic field reversal, Nature, 377, 203209, 1995a.

Glatzmaier, G. A. and P. H. Roberts, A three-dimensional convective dynamo solution with rotating and finitely conducting inner core and mantle, Phys. Earth Planet. Inter., 91, 63-75, 1995 b.

Horiuti, K., A new dynamic two-parameter mixed model for large-eddy simulation, Phys. Fluids, 9, 3443-3464, 1997.

Kageyama, A. and T. Sato, Generation mechanism of a dipole field by a magnetohydrodynamic dynamo, Phys. Rev. E, 55, 4617-4626, 1997.
Kageyama, A., T. Sato, and the Complexity Simulation Group, Computer simulation of a magnetohydrodynamic dynamo. II, Phys. Plasmas, 2, 1421-1431, 1995.

Kono, M. and P. H. Roberts, Recent geodynamo simulations and observations of the geomagnetic field, Rev. Geophys., 40, 1013, doi:10.1029/2000RG000102, 2002.

Matsushima, M., Expression of turbulent heat flux in the Earth's core in terms of a second moment closure model, Phys. Earth Planet. Inter., 128, 137-148, 2001.

Matsushima, M., T. Nakajima, and P. H. Roberts, The anisotropy of local turbulence in the Earth's core, Earth Planets Space, 51, 277-286, 1999.

Olson, P., U. Christensen, and G. A. Glatzmaier, Numerical modeling of the geodynamo: Mechanisms of field generation and equilibration, $J$. Geophys. Res., 104, 10,383-10,404, 1999.

Phillips, C. G. and D. J. Ivers, Spherical anisotropic diffusion models for the Earth's core, Phys. Earth Planet. Inter., 117, 209-223, 2000.

Phillips, C. G. and D. J. Ivers, Spherical interactions of rapidly-rotating anisotropic turbulent viscous and thermal diffusion in the Earth's core, Phys. Earth Planet. Inter., 128, 93-107, 2001.

Phillips, C. G. and D. J. Ivers, Strong field anisotropic diffusion models for the Earth's core, Phys. Earth Planet. Inter., 140, 13-28, 2003.

Roberts, P. H. and G. A. Glatzmaier, Geodynamo theory and simulations, Rev. Mod. Phys., 72, 1081-1123, 2000.

St. Pierre, M. G., On the local nature of turbulence in Earth's outer core, Geophys. Astrophys. Fluid Dynam., 83, 293-306, 1996.

Takahashi, F., J. S. Katayama, M. Matsushima, and Y. Honkura, Effects of boundary layers on magnetic field behavior in an MHD dynamo model, Phys. Earth Planet. Inter., 128, 149-161, 2001.

Zhang, K. and C. A. Jones, The effect of hyperviscosity on geodynamo models, Geophys. Res. Lett., 24, 2869-2872, 1997.

M. Matsushima (e-mail: mmatsush@geo.titech.ac.jp) 